\title{
Metadata standards: The DAM accelerator!
}

\section{Dianne Kennedy}

is the vice president of publishing technologies for IDEAlliance. IDEAlliance is a not-for-profit membership organization that has been a leader in information technology since 1966. IDEAlliance advances core information technologies to develop standards and best practices that enhance efficiency and speed in all facets of content creation, production, management and delivery - digitally and in print. Currently Ms. Kennedy serves as Program Director for the IDEAlliance metadata initiatives including PRISM, DISC and DIM2.

Keywords: digital asset management, metadata standards, Dublin Core, PRISM, Digital Image Management Metadata, Digital Image Submission Criteria, XML, RDF, XMP

Abstract The cost of implementing a digital asset management (DAM) system is never just the cost of the software and hardware. Often the far greater cost in time and dollars is developing an organizational scheme for the assets. But knowing how to leverage industry metadata standards can prove to be a real DAM accelerator! Journal of Digital Asset Management (2006) 2, 134-137. doi:10.1057/palgrave.dam.3650023

\section{IT'S ALL ABOUT METADATA}

According to Marty Lucas in $2000,{ }^{1}$ "Metadata is really an age-old answer to an age-old problem. The problem is, how to get the most out of a stored collection of information." Lucas goes on to say, "Datastores are bigger than ever and so is the problem. A consensus is growing that metadata is the answer. Metadata is often described as 'information about information' but I prefer to think of it as another layer of information simplified, distilled, made orderly — created to help people use an information source."

Of course, today we know that Lucas was right on target. Whether information is collected in a library, a medical filing system or in an editorial office, we still need to know how to file and retrieve that information. Whether we are using file cabinets and file folders or a very sophisticated digital asset management (DAM) system, we need a way to label and organize information. And metadata is that way. Metadata is that information layer that helps us manage and use digital assets.

\section{ALL KINDS OF METADATA}

Dianne Kennedy VP Publishing Technologies, IDEAlliance, Alexandria, VA 22314-2805 USA Tel: +1 6309418197 E-mail: dkennedy@ idealliance.org

\section{So if we need a metadata scheme to organize} our assets, how can we get one? Well of course you could develop your own. You could seek expert help to do so. Or you could look for one that was developed and made freely available for anyone to use. So how do you know what to do? What questions should you ask?

Well, the first question is why do you need metadata? We know you want it to help you manage digital assets, but what does that mean? Do you want metadata to facilitate archive and retrieval? Rights management? Usage management? Can you start with one function (the most important) and build from there? An example is the stated goal of the IEEE (Institute of Electrical and Electronics Engineers) learning object metadata (LOM) project, " "The Learning Object Metadata standards will focus on the minimal set of attributes needed to allow these Learning Objects to be managed, located, and evaluated."

Once you know why you want metadata, you need to decide what kind of metadata fields you need. In other words, how do you want to organize assets? Do you want to organize assets based on their description? Or may be by who created the asset? May be you want to organize assets chronologically. By their cost? Or maybe by their digital format? It turns out that there are many kinds of metadata.

Some common metadata types included are as follows:

- subject matter/descriptive

- price

- digital format 
- rights

- date/time

- links/relationships

- structural.

A rule of thumb in designing a metadata scheme is to have a unique identifier for each asset and multiple paths to reach the asset. Those paths will depend on the type(s) of metadata you include in your design based on your usage scenario. The paths to reach the asset are really dependent on the metadata fields that you define.

\section{WHY USE A METADATA STANDARD?}

There are several obvious reasons for you to jumpstart your project using an established metadata standard. The first reason is that a standard gives you a great starting point. If you can find a standard that fits your business model, you can leverage the hard work of bright people as your beginning point. Many standards have taken years to develop. In part, this is because standards typically have broad industry participation and many ideas and opinions come to the table. Industrywide debates take place about how assets should be organized and why one approach is superior to another.

Let look at the PRISM (Publishing Requirements for Industry Standard Metadata) specification that was developed by IDEAlliance. The PRISM Working Group included publishers, other rights holders, systems integrators, software developers and content aggregators who faced common content application challenges such as reuse of content in multiple media types, rights and contract management, better access to content archives and faster, less expensive exchange and integration of disparate sets of content across the enterprise and with outside business partners. The work on PRISM began in 1999. The first version of the specification was released in 2001. If you look at the acknowledgments page for the specification, ${ }^{3}$ you will see that approximately 50 companies and 70 individuals have participated in this effort. Quite a team to be sure!

Then there is the benefit of industry oversight. Working Groups, such as PRISM, post their specifications for public comment. This brings even more bright minds and expertise to the final metadata design.

Finally, there is the advantage of insights gained by adoption of a standard. If you design your own metadata scheme, your implementation will be the first test. If you base your design on a standard, you have the benefit of improvements that have been made as organizations have learned lessons as they implemented the specification. According to the press release for PRISM Version 1.2, Since the 2002 release of the PRISM 1.1 specification, more and more publishers, integrators and software vendors have recognized its value and incorporated it into their content and information management strategies.

Implementation experience by companies such as Time Inc. led to ideas for new vocabulary terms, and a desire to incorporate PRISM into RSS 1.0 feeds also led to updates to the specification. PRISM 1.2 was developed in direct response to the new requirements of the implementation community. ${ }^{4}$ Version 1.3 of PRISM Specification will be released this fall.

\section{SELECTING AN APPROPRIATE METADATA SPECIFICATION}

The first place to look for a metadata specification that will fit your DAM is within your own industry. Are you interested in managing video or music? Then you will want to look to groups in your industry to see if someone is developing or has developed a metadata specification to meet your business requirements. If you are in the field of education and have multimedia assets, then maybe the LOM project from IEEE is a good fit. If you are a magazine or journal publisher, PRISM may address your needs. If your focus is digital images, there are numerous metadata specifications you might use as a base, including (International Press Telecommunications Council) IPTC, ${ }^{5}$ digital image submission criteria (DISC) metadata specification ${ }^{6}$ or the PRISM digital image management metadata (DIM2) specification. ${ }^{7}$ You need to look at the specifications in terms of their business goals and also in terms of the fields that provide access paths to the data.

Another factor you should consider is the format used by the metadata specification that 


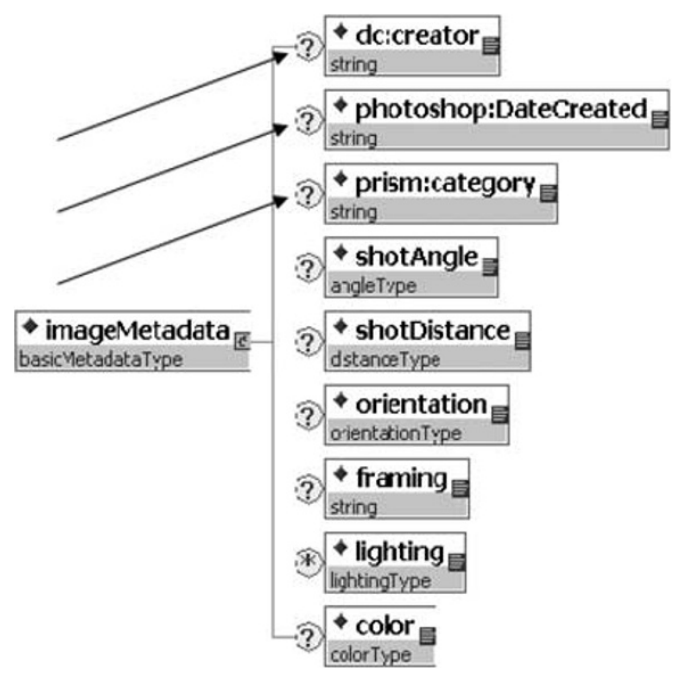

Figure 1: Combining metadata fields from outside namespaces

you are considering. You need to be sure that this metadata format can be handled by the DAM systems you have purchased or are considering. Most metadata specifications recommend a specific format or syntax. In many cases, this is XML (worldwide web consortium extensible mark-up language). ${ }^{8}$ In some cases, an resource definition framework (RDF)/XML syntax is recommended. RDF is the worldwide web consortium resource description framework. ${ }^{9}$ Some specifications define fields but do no recommend a specific syntax. And others, such as those from IDEAlliance, allow for two profiles or two different syntaxes.

\section{MIXING AND MATCHING}

If you study modern metadata specifications, you will find that it is common for one specification to build upon another using a mechanism called "Namespaces." Namespaces are a prefix that can be attached to a metadata field name indicating where it came from. This mechanism lets you combine metadata fields from several specifications when those fields meet your business requirements. Many metadata specifications build on others using this namespace mechanism.

Perhaps, the grandfather of all metadata specifications is the Dublin Core. ${ }^{10}$ Named for Dublin, Ohio, home of the Online Computer Library Center, the Dublin Core Metadata Initiative established a set of metadata to describe electronic resources in a manner similar to a library card catalog. The Dublin Core includes 15 general elements designed to characterize resources. PRISM uses the Dublin Core and its relation types as the foundation for its metadata. All IDEAlliance metadata specifications are built upon Dublin Core as are metadata sets from IPTC and even XMP (extensible metadata platform from Adobe). ${ }^{11}$

The following illustration from the new PRISM DIM2 metadata specification for digital images shows how we have built on other metadata specifications to construct our metadata definition for digital images. Note fields from Dublin Core (dc:), Photoshop (photoshop:) and PRISM (prism:) are used in addition to new elements defined by the DIM2 Working Group (Figure 1).

\section{MAKE IT YOUR OWN}

The likelihood that you can find a metadata standard that will exactly meet all your needs is slim. So in using a metadata standard you must build on the standard and make it your own. There are two major activities in doing this.

First you must realize that it is likely that you will not need all the fields in the specification. Let us look at the DISC metadata specification as an example. This specification was developed by the IDEAlliance DISC Working Group. The goal of the specification is to enable magazines to require standard metadata from photographers at the time of image submission to enable the image to easily merge into the digital workflow. In developing the DISC metadata specification, it became clear that the different digital workflows employed by magazine publishers meant that different metadata would be required from magazine to magazine. Therefore the DISC Metadata Specification includes 16 metadata fields that are an aggregation of the requirements of the magazine industry. Each magazine will take a subset of these fields that fit their workflow. And it is likely that whatever specification you choose as a foundation, you will want to take a subset of the fields as well.

A second consideration in making a metadata specification your own is to figure out what is missing. One might expect that you can leverage 80 or 90 per cent of the metadata fields to meet your needs from outside standards. But it is 
quite likely that you will find business requirements that demand you create new paths to your assets by defining metadata fields. This is expected and most organizations do this. Remember that using the standard still has saved you a lot of time and money. You are certainly doing far less analysis than if you started from a blank sheet of paper!

\section{WHAT IF THERE IS NO STANDARD FOR ME?}

Depending on your business, you may search and not find a standard upon which to base the organization of your DAM system. What do you do then? Well, you are back to designing your own with the help of an expert consultant. Or are you?

If there are others in your business arena, they most likely are or will be facing much the same challenge as you are. So why not work together? Your development team will suddenly be far broader and have more industry expertise. And groups of potential DAM buyers can often gain attention from technologists on the vendor side that any individual company cannot. In other words, why not develop your own metadata standard? This is exactly how PRISM, DISC, and DIM2 metadata specifications came to be. IDEAlliance is currently chartering new working groups to develop metadata specifications to jump start implementation of DAM systems for 2006. Maybe yours will be one of them.

\section{CONCLUSION}

Developing your own organizational scheme for digital assets can be a lonely business. A much smarter idea is to base your work on existing metadata standards. These can accelerate the process of implementing a DAM and help error proof your design.

\section{References}

1 Marty, L. (1999) "Demystifying metadata", available at: http://mappa.mundi.net/trip-m/metadata/.

2 Learning Technology Standards Committee. Working Group 12. (2002) "Learning Object Metadata Specification", available at: http://ieeeltsc. org/wg12LOM/.

3 PRISM Working Group. (2005) "PRISM Introduction," Vol. 1.3, available at: http://www. prismstandard.org.

4 PRISM Publishing Industry Metadata Specification Version 1.2 Released (2005) available at: http:// www.prismstandard.org/news/2005/0104.asp.

5 International Press and Telecommunications Council, IPTC Metadata for XMP (2005) available at: http://www.iptc.org/IPTC4XMP/.

6 Seybold Report, Vol. 5, No. 10, 17 August 2005.

7 PRISM Digital Image Management Working Group, DIM2. Specification, available at www. prismstandard.org (2004).

8 Tim, B., Paoli, J. and Sperberg-McQueen, C.M. "Extensible Markup Language (XML)" (2004) (eds.), available at: http://www.w3.org/TR/REC-xml.

9 Ora, L. and Swick, R.R. "Resource Definition Framework (RDF) Model and Syntax Specification" (2002) available at: http://www.w3.org/TR/ REC-rdf-syntax.

10 Dublin Core Metadata Element Set, Version 1.1: Reference Description" (2005) available at: http:// purl.org/dc/documents/rec-dces-19990702.htm.

11 Adobe Systems, XMP Specification" (2004) available at: http://partners.adobe.com/public/developer/ en/xmp/sdk/xmpspecification.pdf.

12 Dublin Core Metadata Element Set, Version 1.1" (2004) available at:http://dublincore.org/documents/ dces/. 